\title{
ACCIONES EDUCATIVAS INNOVADORAS PARA EL DÍA INTERNACIONAL DE LA MUJER Y LA NIÑA EN LA CIENCIA EN INFANTIL Y PRIMARIA
}

\author{
Yolanda Sevilla Vera \\ Departamento de Psicología, Área de Psicología Evolutiva y de la Educación \\ Facultad de Educación de Toledo \\ Universidad de Castilla La Mancha, España \\ yolanda.sevilla@uclm.es \\ Natalia Solano Pinto \\ Departamento de Psicología, Área de Psicología Evolutiva y de la Educación \\ Facultad de Educación de Toledo \\ Universidad de Castilla La Mancha, España \\ natalia.solano@uclm.es \\ Dunia Garrido \\ Departamento de Psicología, Área de Personalidad, \\ Evaluación y Tratamiento Psicológico \\ Facultad de Ciencias de la Salud, Talavera de la Reina \\ Universidad de Castilla La Mancha, España \\ dunia.garrido@uclm.es \\ Raquel Fernández Cézar \\ Departamento de Matemáticas, Área de Didáctica \\ Facultad de Educación de Toledo \\ Universidad de Castilla La Mancha, España \\ raquel.fcezar@uclm.es
}

Recepción Artículo: 13 marzo 2020

Admisión Evaluación: 30 marzo 2020

Informe Evaluador 1: 15 abril2020

Informe Evaluador 2: 19 abril 2020

Aprobación Publicación: 20 abril 2020

\section{RESUMEN}

Antecedentes. El conocimiento científico y la igualdad de género son cruciales para alcanzar los Objetivos de Desarrollo Sostenible. Las iniciativas de innovación educativa son las que acercan estos a toda la comunidad educativa, inspirando y promoviendo la participación de las mujeres y las niñas en la ciencia, eliminando la brecha de género en el ámbito científico y otros sectores.

Objetivos. Fomentar la organización de actividades que promuevan el desarrollo científico y tecnológico en la infancia con la colaboración de la familia, desarrollando una mirada curiosa y transformadora.

Metodología. La muestra estuvo formada por 182 participantes (85 niñas y 97 niños) de infantil y primaria; 16 miembros del profesorado y familias.

Instrumento y procedimiento. En el marco del "Día Internacional de la mujer y la niña en la ciencia", se llevó acabo en el CEIP un amplio programa de actividades acerca de la ciencia y conocer la importancia de la mujer 


\section{ACCIONES EDUCATIVAS INNOVADORAS PARA EL DÍA INTERNACIONAL DE LA MUJER Y LA NIÑA EN LA CIENCIA EN INFANTIL Y PRIMARIA}

en esta materia, intentando sensibilizar y fomentar el pensamiento científico y tecnológico en toda la comunidad educativa.

Resultados. El alumnado de los cursos más altos manifestaba una desigualdad de género y en algunos casos les costaba identificar dicha desigualdad en el desempeño de tareas, responsabilidades y profesiones, observándose lo contrario en los más pequeños.

Conclusiones. Los datos arrojan la necesidad de seguir fomentando este tipo de actividades y programas.

Palabras clave: innovación docente; educación, mujer y ciencia; acciones educativas

\section{ABSTRACT}

Innovative educational actions for the international day of women and girls in science at infant and primary level. Background. Science and gender equality are crucial for achieving the Sustainable Development Goals. Educational innovation initiatives are those that bring these goals closer to the entire educational community, inspiring and promoting the participation of women and girls in science, eliminating the gender gap in science and other sectors.

Objectives. To promote the organization of activities that foster scientific and technological development in children with the collaboration of the family, developing a curious and transforming glance.

Methodology: The sample was made up of 182 students ( 85 girls and 97 boys) from preschool and primary schools; 16 teachers and families.

Instrument and procedure. In the framework of the "International Day of the woman and the girl in the science", it is carried out in the school an ample program of activities about the science, to know the importance of the woman in this field, trying to generate awareness and to promote the scientific and technological thought in all the education community.

Results. The students in the higher courses showed gender inequality and, in some cases, they found it difficult to identify that in the performance of tasks, responsibilities and professions, while the opposite was observed in the youngest children.

Conclusions. The data showed the need to continue promoting these kinds of activities and programs.

Keywords: teaching innovation; education, women and science; educational actions

\section{INTRODUCCIÓN}

En los últimos tiempos es de interés para todas las comunidades educativas trabajar sobre la importancia de la ciencia y la igualdad de género con el fin de poder afrontar los desafíos del siglo XXI, aprovechando todo el potencial que tiene el ser humano sin sesgo alguno. Es vital para alcanzar los Objetivos de Desarrollo Sostenible (ODS), y se pone en valor el esfuerzo realizado en esta dirección por la comunidad internacional, incentivar y promover y visibilizar la participación de la mujer y la niña en la ciencia. Ese esfuerzo requiere el impulso de acciones como el Día internacional de la mujer y la niña en la ciencia, que pretende poner en valor iniciativas orientadas a poner fin al desequilibrio de género en esta disciplina (Guterres, 2019).

En 2015, Ios líderes mundiales acordaron cumplir para 2030 con el Objetivo de Desarrollo Sostenible número 9 (Industria, Innovación e Infraestructuras). Los países de todo el mundo se comprometieron a desarrollar infraestructuras fiables, sostenibles, y de calidad, para conseguir una infraestructura resistente, y así promover una industrialización inclusiva, sostenible e innovadora.

Como afirman autores como Brea (2020), la sociedad tiene que saber por qué es importante la ciencia y en este proceso tenemos que ser partícipes hombres y mujeres. Es por ello que todo contenido didáctico, debe dar la máxima visibilidad a los logros alcanzados por las mujeres científicas que en la actualidad trabajan a nivel internacional, concienciado a las comunidades educativas y a la sociedad del importante papel que tienen las mismas en las disciplinas STEAM (Science, Technology, Engineering, Arts and Maths).

Por esta razón, la ciencia, la tecnología y la ingeniería son algunas de las materias en las que se fundamenta nuestro estilo de vida, y su estudio debe ocuparnos. No deben asustarnos las nuevas normas, la innovación y 
nuevas fórmulas. La tecnología suple cualquier nexo de comunicación y ayuda a llevar situaciones complicadas y adversas con más facilidad. Por lo tanto, nuestras comunidades educativas han de estar preparadas para cualquier cambio (Sevilla y Solano, 2020), buscando la metodología que fomente la creatividad, el emprendimiento, la imaginación y otras habilidades en cualquier cambio científico, según afirma Olga Vakula (2020), que es la Oficial adjunta de información nuclear en el Organismo Internacional de Energía Atómica (OIEA).

Cuando se comenzó con el diseño del programa de acciones educativas innovadoras para el Día Internacional de la Mujer y la niña en la Ciencia, surgió la pregunta, ¿cuál era la razón por la que se dedicaba un día internacional?

La respuesta ha sido contemplada por las Naciones Unidas como un tema central, siendo la igualdad entre los géneros y el empoderamiento de las mujeres y las niñas una contribución decisiva, no solamente al desarrollo económico del mundo, sino también una pieza fundamental en el progreso de todos los objetivos y metas de la Agenda 2030 para el Desarrollo Sostenible.

Sería el 14 de marzo de 2011, cuando se celebraba la Comisión de la Condición Jurídica y Social de la Mujer, cuando tuvo lugar la aprobación del informe correspondiente al 55ํㅜ período de sesiones. Dicho informe contiene las conclusiones sobre el acceso y la participación de la mujer y la niña en la educación, la capacitación y la ciencia y la tecnología, incluida la promoción de la igualdad de acceso de la mujer al pleno empleo y a un trabajo digno. Esto puede pasar desapercibido y ser obsoleto o parecer de otra época, pero no está tan lejos de la actualidad, ni de la educación del día a día.

El 20 de diciembre de 2013, la Asamblea General aprobó la resolución relativa a la ciencia, la tecnología y la innovación para el desarrollo. De esta forma, reconoció que el acceso y la participación plenos y en condiciones de igualdad en la ciencia, la tecnología y la innovación para las mujeres y las niñas de todas las edades son imprescindibles para lograr la igualdad entre los géneros y el empoderamiento de la mujer y la niña.

En la Asamblea General fue declarado el 11 de febrero Día Internacional de las Mujeres y las Niñas en la Ciencia en reconocimiento al papel clave que desempeñan las mujeres en la comunidad científica y la tecnología. Así, el 22 de diciembre de 2015 se aprobó una resolución en la que justificaba la proclamación de este Día Internacional y alababa las iniciativas llevadas a cabo de la Organización de las Naciones Unidas para la Educación, la Ciencia y la Cultura (UNESCO), la Entidad de las Naciones Unidas para la Igualdad de Género y el Empoderamiento de las Mujeres (ONU Mujeres), la Unión Internacional de Telecomunicaciones (UIT) y otras organizaciones competentes para apoyar a las mujeres científicas y promover el acceso de las mujeres y las niñas a la educación, la capacitación y la investigación en los ámbitos de la ciencia, la tecnología, la ingeniería y las matemáticas. Actualmente las denominamos disciplinas o ramas de la ciencia STEAM (Ciencia, Tecnología, Ingeniería, Artes y Matemáticas).

En el Día Internacional de las Mujer y las Niña en la Ciencia se realiza un cambio en la narrativa predominante, se celebra un liderazgo en la innovación y se solicita una actuación para derrumbar las barreras que todavía se encuentran, con la intención última de que no se circunscriba a ese día, sino que se extienda a todo el año. España ha sido un país en el que esta iniciativa comenzó a desarrollarse desde 2016, teniendo en la actualidad un peso muy importante a nivel mundial (www.11defebrero.org )

Las mujeres y las niñas constituyen la mitad de la población mundial y por consiguiente la mitad de su potencial. La igualdad de género, además de ser un derecho humano fundamental, es imprescindible para lograr sociedades pacíficas, con pleno potencial humano y desarrollo sostenible.

En la misma línea, durante la Presidencia Española de la Unión Europea en 2010 el Consejo de Competitividad, donde se reúnen los ministros europeos de investigación e innovación, se adoptó un importante acuerdo para apoyar a las mujeres en la ciencia y promover el cambio estructural a través de la modernización de las instituciones científicas. En este acuerdo por un lado se recogía las recomendaciones del documento Gender and Research Beyond 2009 del Grupo de Helsinki, y, por otro, instaba a la Comisión a adoptar una Comunicación sobre Cambio Estructural, es decir, una recomendación dirigida a los estados miembros para que actuaran en este sentido. 


\section{ACCIONES EDUCATIVAS INNOVADORAS PARA EL DÍA INTERNACIONAL DE LA MUJER Y LA NIÑA EN LA CIENCIA EN INFANTIL Y PRIMARIA}

Apoyados en estos documentos se realiza la propuesta y organización de estas jornadas en el centro educativo, donde la pretensión no es solamente una propuesta de actividades, sino un conjunto de acciones educativas innovadoras con intención de permear a la comunidad educativa y su idiosincrasia, tal y como enfatizan Vázquez y Manassero (2007) al revisar las actitudes en la educación científica.

A diario, se observa en el aula materiales curriculares y recursos que se manejan, donde se aprecian, matices de sesgo por género. Es habitual encontrar este sesgo en los libros de texto (Cobano-Delgado Palma y Terrón Caro, 2008; Del Río Mayordomo, 2019). En general, en la escuela se hace poca referencia a las mujeres científicas y su aportación a la ciencia, salvo cuando se trata de la celebración de efemérides. Por tanto es de interés e importancia, el esfuerzo conjunto, constancia y motivación en el mismo para contrarrestar esta situación transmitiendo igualdad de género desde la infancia, la base de nuestra pirámide educativa, para que se conozca y se descubra el conocimiento de manera completa y sin sesgos de género. Es de vital importancia despertar la curiosidad, parte inherente de la persona, para que la imaginación y la creatividad fluya en nuestras alumnas y alumnos. A ello ayudan iniciativas como la del 11 de febrero, siendo esta actividad, una iniciativa del presente que dará paso a la construcción del futuro. Estas iniciativas en las se reconoce el papel de las mujeres en la comunidad científica y la tecnología, que contribuyan a empoderar a las mismas, son aún más necesarias en una zona rural, donde los estereotipos de género están aún más enraizados. Estudios recientes prueban que la mente infantil es muy permeable al conocimiento y al método científico (García, 2014), pues nuestro cerebro aprende experimentando e hipotetizando. Frente a las metodologías tradicionales, que constituyen contextos educativos estáticos, pocos innovadores, donde no ven más allá de meras repeticiones, rutinas sin sentido y supeditados al mundo de un único recurso los libros de texto, estas nuevas metodologías de enseñanza, que ponen en valor la cultura científica sin sesgo de género, fomentan un aprendizaje basado en la creatividad y la curiosidad.

Un acercamiento básico al saber científico puede establecer una base sólida para futuros aprendizajes y proporcionar al niño expectativas que le hagan interesante la actividad (Caravaca, 2010). Además, según afirman autores como Po (2003), el aprendizaje de las ciencias no debe ser concebido solamente en términos cognitivos, se debe tener en cuenta la interacción y el desarrollo afectivo.

Por los motivos anteriores, se pretende fomentar la organización de actividades que promuevan el desarrollo científico y tecnológico en la infancia con la colaboración de la familia, desarrollando una mirada curiosa y transformadora. Promocionar la educación científica como soporte esencial para la defensa de un currículo co-educativo, basado en la innovación y metodología STEAM, e impulsar la educación emocional en un ambiente científico y que sea el desarrollo afectivo complemento indispensable del desarrollo cognitivo, logrando una actitud científica positiva en igualdad de género. $\mathrm{E}$

\section{MÉTODO}

Este trabajo, se llevó a cabo a través del diseño de un programa de acciones educativas innovadoras en el marco del "Día Internacional de la mujer y la niña en la ciencia".

Fue desarrollado dentro del ámbito educativo, en horario lectivo, mediante clases colaborativas y activas con el propósito y requisito de la implicación de toda la comunidad educativa, tomando como base la innovación educativa.

El proyecto se enmarca en la programación general anual para el curso escolar donde se plantean las competencias que tienen que desarrollar y adquirir, a través de las actuaciones diseñadas.

Igualmente, esta iniciativa se une a la plataforma del 11defebrero.org, en la que se pretende contribuir a cerrar la brecha de género que actualmente existe en el ámbito científico en el ámbito español. Igualmente, estas acciones educativas pretenden una mejora en el ámbito científico educativo desde la perspectiva de género en nuestro centro y desde la base del sistema educativo, la etapa de educación infantil y primaria.

Para ello el centro educativo ha preparado un programa de actividades para acercarse a la ciencia y conocer la importancia de la mujer en esta materia, intentando especialmente sensibilizar a los más pequeños de la casa 
en compañía de sus familias. Se trabaja dentro de un modelo inclusivo con la apertura del centro a toda la comunidad educativa a través del proyecto STEAM y el programa Carmenta.

\section{DESCRIPCIÓN DEL PROYECTO}

\section{Participantes}

Los participantes fueron 182 alumnos (85 niñas y 97 niños) de los cursos de 3, 4 y 5 años de educación infantil y de $1^{0}$ a $6^{0}$ de primaria, con edades entre 3 y 12 años. Se contaba con la participación de toda la comunidad educativa, profesorado y familias. Se trata de una zona rural al sur de la provincia de Cuenca (Las Mesas), de la Comunidad de Castilla La Mancha.

\section{Desarrollo del proyecto}

La intervención pedagógica tuvo un carácter práctico y procesual comunicativo. Se implementó durante cuatro semanas, comprendidas desde el 11 de febrero y el 13 de marzo, con un total de 30 sesiones de 45 minutos cada una. A cargo de la intervención estuvo la primera autora del artículo, acompañada por otra docente, por lo que las evidencias fueron recogidas y anotadas con más detalle y unificación de criterio y el seguimiento fue más exhaustivo.

Primeramente, tuvo lugar la creación, planificación y difusión (Figura 1) del amplio programa, con la colaboración de varias instituciones. Se perseguía sensibilizar y fomentar el pensamiento científico y tecnológico, fundamental para el alumnado de estas zonas con especial incidencia de la despoblación (Estrategia ITI, Inversión Territorial Integrada para el desarrollo de zonas con despoblamiento y declive socioeconómico en Castilla - La Mancha).

Posteriormente, se realizaron las sesiones de experimentos en el laboratorio, talleres de realidad virtual, trabajos de documentación e investigación, charlas-coloquio realizando no solamente clases expositivas con apoyo digital, sino con una metodología activa y participativa.

El proyecto se desarrolló bajo el lema "Ciencia a conciencia" y contó con la colaboración de varias entidades e instituciones públicas y privadas, como UCLM, Forum CLM, Guardia Civil, Instituto de la Mujer, así como el ayuntamiento y empresas de la localidad, donde el alumnado de primaria actuaría como divulgador científico, adoptando el papel de profesor, compartiendo así su experiencia de forma directa y recibiendo formación a su vez.

Durante varias sesiones, los alumnos trasladaron lo aprendido a la comunidad educativa, a través de varios experimentos de laboratorio y con realidad virtual, diseñados por ellos mismos, con la supervisión del profesor, de la mano del proyecto STEAM y programa Carmenta. Además, se realizaron talleres basados en mujeres célebres, así como, en aquellas novedades que hoy en día son de interés general para la comunidad educativa. Para la semana final del proyecto, realizaron una conexión con las futuras maestras y futuros maestros (alumnado de la Facultad de Educación de Toledo de la Universidad de Castilla La Mancha) mediante video conferencia, y se tuvo la oportunidad de exponer cómo se estaba trabajando en el centro educativo por parte de los más pequeños. Ambos grupos de estudiantes interactuaron compartiendo experiencias reales de innovación educativa que actualmente se está llevando a cabo en el aula. Estas experiencias son significativas para los futuros maestros, pues sus percepciones sobre las aulas no siempre coinciden con la realidad. Se recogieron evidencias de video y audio de las opiniones emitidas.

Todo el proyecto se integró dentro de las programaciones diseñadas para cada nivel y curso escolar, como documentos vivos en continua revisión. Las sesiones de videoconferencia con el alumnado de la Facultad de educación de Toledo se realizaron en turno de mañana. Las sesiones con las familias tuvieron lugar por la mañana y una sesión por la tarde aprovechando las horas de atención tutorial. En dichas sesiones con las familias, fueron los propios niños los que ejercieron de tutores con sus familias, con la participación de padres y abuelos. Se enfatiza este aspecto por la importancia de las abuelas, mujeres que forman parte del núcleo familiar y, muchas 


\section{ACCIONES EDUCATIVAS INNOVADORAS PARA EL DÍA INTERNACIONAL DE LA MUJER}

Y LA NIÑA EN LA CIENCIA EN INFANTIL Y PRIMARIA

veces, sirven de referencia adulta presencial en la realización de tareas. Es importante este aspecto, que seguiremos desarrollando e investigando. En las sesiones organizadas en el centro educativo, cada uno de los experimentos son compartidos vía streaming por el profesorado, dando opción a que otros niveles puedan conectarse para participar de la sesión que los compañeros están desarrollando. La temporalización para esta actividad, se desarrolló durante los meses de febrero y marzo, en horario de mañana y de tarde. El lugar elegido para la muestra de estos trabajos fue el centro educativo, que ha sido equipado con todo lo necesario para que los alumnos y alumnas puedan participar, interactuar, aprender y divertirse en compañía de sus familias con la guía del profesorado y optimizando los recursos.

\section{DISCUSIÓN}

El objetivo del presente trabajo fue poner en marcha un programa de intervención didáctica, destinado a mejorar las actitudes científicas apoyado en metodología STEAM y programa Carmenta, dirigido a toda la comunidad educativa de las etapas de infantil y primaria de un centro escolar público situado en una zona rural de Castilla La-Mancha (Las Mesas-Cuenca).

La idea de promocionar la educación científica en alumnos de las etapas de infantil y primaria, conllevó el desarrollo de un programa de acciones educativas innovadoras, lo cual supuso, un reto para el centro, requiriendo una muy buena coordinación por parte del profesorado inter-etapas, inter-nivelar e interdisciplinar, con la máxima participación y colaboración de las familias, con el soporte de las nuevas tecnologías e innovación educativa y la totalidad del claustro de profesores.

Debemos de tener en cuenta que cuando se lleva a cabo un proyecto de innovación en un centro educativo, es requisito esencial la participación mínima de al menos el 50 por ciento del profesorado. Por tanto, en un programa corto e intenso como fue este, el grado de implicación conllevaría un porcentaje mayor, siendo indispensable para lograr un resultado óptimo y de un mayor impacto en la comunidad educativa.

Este planteamiento sirvió de punto de partida para la puesta en práctica de experiencias de aula compartidas, prácticas de laboratorio, realidad virtual, iniciación en diferentes estrategias metodológicas alejadas de un contexto estático, donde una propuesta es capaz de generar actividad, aprendizaje e interacción en el momento, sin necesidad de crear escenarios planificados y con cierta flexibilidad dentro de la coordinación necesaria. Podríamos decir que se dan relaciones en la comunidad similares a la composición de un cuadro "cubista", donde el profesor induce, acompaña, y en el momento, provoca el nacimiento de una situación de aprendizaje. Este mismo escenario se trasladaría a las familias, donde el rol de profesor lo asumiría el alumnado, y el profesor pasaría a un segundo plano, sirviendo de soporte y apoyo.

Este engranaje no es posible sin la educación emocional: consiguió estimular un clima y ambiente científico, el cual contribuyó al desarrollo afectivo como complemento indispensable del desarrollo cognitivo, sin obviar una actitud científica positiva en igualdad de género.

Sería necesario realizar estudios para conocer las actitudes científicas, ya que alumnos de cursos más bajos, como los de ed. infantil, primero y segundo de primaria, de edades entre los 3 y 8 años, no manifestaron tanta diferencia de género como sus compañeros de cursos más altos. Posiblemente la visión de género de estos últimos venga influenciada por los estereotipos transmitidos por los personajes de moda, sus ídolos, medios de comunicación y por los trabajos de sus familiares. Si bien es cierto que, al desarrollarse en el aula metodologías más activas e innovadoras, su percepción se hace más abierta y menos sesgada. Por esta razón parece que esta propuesta inclusiva, pues no se realiza una diferenciación inter-etapas, contribuye a disminuir el sesgo de género en la escuela, en particular en la ciencia, y deja abierta una línea de investigación atendiendo al desarrollo evolutivo del alumnado más que a su edad cronológica.

El proceso de aprendizaje no se limita únicamente a la adquisición de conocimientos, sino que comprende de forma integrada todos los componentes del currículo, en el que se incluyen los valores. Estos, por tanto, no se desarrollan en el vacío sino que deben estar fundamentados en los conocimientos relevantes (Pereiro y Jiménez, 2011), entre los que se encuentra el conocimiento científico. Este conocimiento en su versión tradicional 


\section{CRECIMIENTO PSICOLÓGICO Y AFRONTAMIENTO DE LA MADUREZ}

evidencia diferencias de género, que pueden ser contrarrestadas con la educación STEAM que fomenta la inclusividad en todos los ámbitos, también en género. Dado que las diferencias de género se manifiestan desde edades tempranas, el inicio en la educación STEAM desde estas fomentará la minimización del sesgo de género, y, en prospectiva, que este disminuya en edades superiores si se continua este tipo de educación en los centros escolares.

Autores como Vázquez y Manassero (2008) reportan que se ha producido un declive de las actitudes hacia las ciencias de los estudiantes. Nuestra experiencia se alinea con ellos en tanto que se produce una pérdida de interés en las materias científicas en los cursos más altos de primaria, que serían los niveles de quinto y sexto, lo cual puede afectar en una disminución de este interés por participar en estudios avanzados en cursos posteriores. En cuanto al género, hacen referencia a que cuando se tiene que participar en un concurso de matemáticas pocas veces señalan a las niñas. Sin embargo, cuando se realiza una prueba objetiva y sus resultados son iguales, 0 incluso superiores los de las niñas, es cuando el profesorado y ellas mismas asumen su capacidad de participación. Su proyecto en un futuro, siempre se inclina hacia otras disciplinas y ámbitos no tan relacionados con STEAM. Esta situación tan poco igualitaria e inclusiva es un indicador inquietante para la educación científica, y sugiere la necesidad de la introducción de programas y metodologías innovadoras en las comunidades educativas, como la STEAM. Esta necesidad deviene más importante si cabe en las zonas rurales, por el riesgo actual de despoblación y la incidencia de los estereotipos de género. Para intentar revertirlo es fundamental la implementación de programas como el aquí presentado, en el que se vivencia de forma directa y real estos avances en la educación, enfatizando la perspectiva de género, que puede servir de impulso para estimular las actitudes científicas y emprendedoras de los ciudadanos de zonas ITI.

\section{CONCLUSIONES}

Toda actividad de sensibilización es poca para lograr comprender la implicación que conlleva conseguir una sociedad más justa e inclusiva, siendo un desafío ineludible del sistema educativo, y dentro de este, de la educación científica formal.

A la inclusión total y en cuanto al género contribuyen acciones como la presentada en este trabajo, basada en la educación STEAM. Este promueve la incorporación a nuestros contextos diarios de todo aquello que trabaja en pro de la equidad, para alcanzar un horizonte igualitario. El sistema educativo ha sido y deber ser un importante motor de cambio, de ahí que toda acción educativa innovadora redunde en la consecución de cambios en la comunidad educativa.

La preocupación que nos aborda en el planteamiento de este proyecto de centro, que involucra a toda la comunidad educativa, es evidenciar que el futuro en constante cambio nos reclama la formación y actualización permanente para poder seguir el ritmo de los cambios sociales. Pero en esos cambios es importante no perder la perspectiva y velar por la equidad e inclusión educativa, eliminando cualquier barrera 0 brecha de género y digital que se detecte en nuestros centros. 
Figura 1: Cartel para difusión de la actividad y programa.

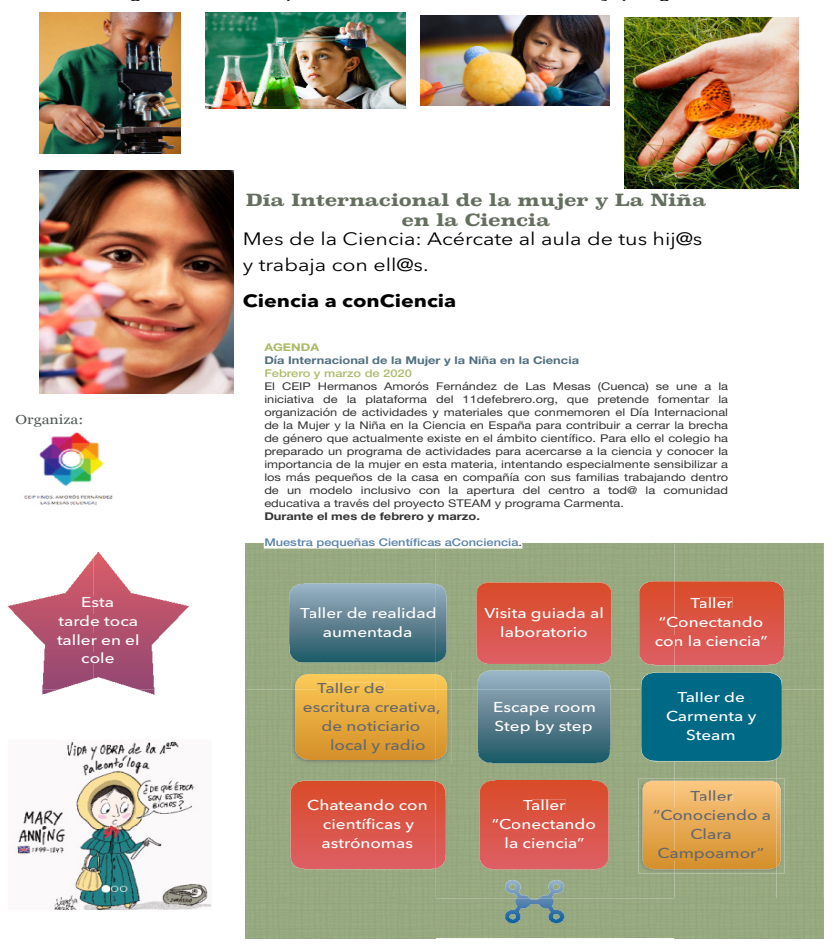

\section{REFERENCIAS BIBLIOGRÁFICAS}

Brea, 0. (2020). Filosofía de la ciencia y educación científica. Innovación y

Educativas, 45, 1-9.

Caravaca, I. (2010). Conocimiento del entorno: acercamiento infantil al saber científico. Innovación y Experiencias Educativas, 36, 1-16.

Cobano-Delgado Palma, V., \& Terrón Caro, Mำ Teresa. (2008). El papel de las mujeres en las ilustraciones de los libros de texto de educación primaria. Foro de Educación(10), 385-400.

Del Río Mayordomo, M. (2019). Las imágenes en los libros de texto de Educación Primaria: ¿Son equitativas respecto al género? (Trabajo fin de máster). Universidad de Castilla La Mancha, Toledo, España.

Domingo, J. (2013). Un marco crítico de apoyo para ubicar y redireccionar experiencias innovadoras en Educación: Comprensión y Transformación. Tendencias Pedagógicas 21, 9-28.

Gairín, J. (2007). La gestión de procesos y del conocimiento. En curso de Asesores de Centros Educativos. Madrid: CENICE- Instituto Superir de Formación del Profesorado.

Gairín, J. (2010). Los Directivos como agentes de cambio en las organizaciones educativas. Documento de trabajo. Universidad autónoma de Barcelona.

Garde, J.J. (2020). 11 de febrero; Día Internacional de las Mujeres y las Niñas en la Ciencia. Ciudad Real. Disponible en https://www.miciudadreal.es/2020/02/10/11-de-febrero-dia-internacional-de-las- mujeresy-las-ninas-en-la-ciencia/. Consultado el 10 de de febrero, 2020.

Consultado el 28 de Marzo, 2020. 
Fundación Española de Ciencia y Tecnología (2005). Evaluación Nacional de Actitudes y Valores hacia las Ciencias en entornos educativos. Madrid: FECYT.

Guterres, A. (2019). ONU. Nueva York. Disponible en https://news.un.org/es/story/2019/03/1452401. Consultado el 1 de Abril, 2020.

Pro A. (2003). La construcción del conocimiento científico y los contenidos de ciencias. Enseñar ciencias, 3354.

Vakula, 0. (2020). Nueva York. Día Internacional de la Mujer y la niña en la Ciencia. ONU. NuevaYork. Disponible en https://www.un.org/es/obsevances/women-and-girls-in-science-day/day/background. Consultado el 23 de Marzo, 2020.

Organización de las Naciones Unidas para la Educación, la Ciencia y la Cultura (2015). A Complex Formula: Girls and Women in Science, Technology, Engineering and Mathematics in Asia. Disponible en https://bangkok.unesco.org/content/complex-formula-girls-and-women-science-technology-engineeringand-mathematics-asia. Consultado el 24 de Marzo, 2020.

Parlamento Europeo y Consejo de la Unión Europea (2006). Recomendación 2006/962/CE del Parlamento Europeo y del Consejo, de 18 de diciembre de 2006, sobre las competencias clave para el aprendizaje permanente [Diario Oficial L 394 de 30.12.2006]. Recuperado de: http://www.mecd.gob. e s/d ctm/ministerio/educacion/mecu/mecu-europa/eqfrecom20080423.pdf?documentld=0901e72b806a600e

Sevilla, Y.; Solano, N. (2020). Inclusión educativa de la mano de Steam y las nuevas tecnologías. Revista de educación e inspección: Supervisión 21, 55. Disponible en https://supervision21.usie.es/wpcontent/uploads/sites/2/2020/03/SP21-55-Art\%C3\%ADculo-Inclusi\%C3\%B3n-educativa-de-manos-dela-STEAM-Sevilla-Vera. Consultado 23 de marzo, 2020.

Vázquez, A.; Massanero, M. A. (2007). En defensa de las actitudes y emociones en la educación científica (I): Evidencias y argumentos generales. Revista Eureka sobre Enseñanza y Divulgación de las Ciencias, 4 (2), 246-271.

Vázquez, A.; Manassero, M. A. (2007). En defensa de las actitudes y emociones en la educación científica (II): Evidencias empíricas generales derivadas de la investigación. Revista Eureka sobre Enseñanza y Divulgación de las Ciencias, 4 (3), 417-441.

Vázquez, A.; Manassero, M. A. (2008). El declive de las actitudes hacia la Ciencia de los estudiantes: un indicador inquietante para la educación científica. Revista Eureka sobre Enseñanza y Divulgación de las Ciencias, 5(3), 274-292. 
